\title{
Experimental evaluation does not reveal a direct effect of microRNA from the callipyge locus on DLK1 expression
}

Huijun Cheng ${ }^{1,2}$, Xuewen X $u^{1,2}$, Tracy Hadfield ${ }^{3}$, Noelle Cockett ${ }^{3}$, Carole Charlier ${ }^{1}$, Michel Georges ${ }^{1}$ and Haruko Takeda ${ }^{1 *}$

\begin{abstract}
Background: Polar overdominance at the ovine callipyge (CLPG) locus involves the post-transcriptional trans-inhibition of $D L K 1$ in skeletal muscle of CLPG/CLPG sheep. The abundant maternally expressed microRNAs (miRNAs) mapping to the imprinted DLK1-GTL2 domain are prime candidate mediators of this trans-effect.

Results: We have tested the affinity of 121 miRNAs processed from this locus for DLK1 by co-transfecting COS1 cells with a vector expressing the full-length ovine DLK1 with corresponding mimic miRNAs. None of the tested miRNAs was able to down regulate DLK1 to the extent observed in vivo.
\end{abstract}

Conclusions: This suggests that other factors, with or without these miRNAs, are involved in mediating the observed trans-effect.

Keywords: Callipyge, Muscle development, Polar overdominance, DLK1, MicroRNA, Trans-effect, Post-transcriptional gene regulation, Reporter assay

\section{Background}

The callipyge phenotype is a muscular hypertrophy that is exclusively expressed by heterozygous sheep inheriting the CLPG mutation from their sire (genotype $+{ }^{\text {Mat }} / C L P G^{\text {Pat }}$ ). This non-Mendelian mode of inheritance with parent-oforigin effect is referred to as polar overdominance [1,2]. The underlying increase in the proportion and size of fast twitch muscle fibers is thought to be caused by ectopic expression of DLK1 and/or PEG11 (also known as RTL1) protein in skeletal muscle of $+{ }^{M a t} / C L P G^{P a t}$ animals. This ectopic expression results from the inactivation - by the CLPG A to G point mutation - of a muscle-specific silencer element that post-natally downregulates the expression of DLK1 and PEG11 in cis [3-6]. CLPG ${ }^{\mathrm{Mat}} /+^{\mathrm{Pat}}$ animals do not express the phenotype because DLK1 and PEG11 genes are imprinted and only expressed from the paternal allele. It is thought that CLPG/CLPG animals do not express the phenotype because of the additional ectopic

\footnotetext{
* Correspondence: htakeda@ulg.ac.be

'Unit of Animal Genomics, GIGA-R \& Faculty of Veterinary Medicine, University of Liège (B34), 1 Avenue de l'Hôpital, 4000 Liège, Belgium Full list of author information is available at the end of the article
}

expression of non-coding RNAs (ncRNAs) that are (i) imprinted and expressed from the maternal allele, (ii) controlled in cis by the same muscle-specific silencer element, and (iii) post-transcriptionally down-regulating DLK1 and PEG11 in trans [7]. Maternally expressed ncRNA genes in the CLPG locus include four long ncRNAs (IncRNA) (GTL2, anti-PEG11, RIAN and MIRG) as well as 110 and 47 embedded miRNAs and C/D snoRNAs, respectively [8-10]. For PEG11 the trans-inhibition results from RNAinduced silencing complex (RISC)-mediated cleavage of PEG11 transcripts by at least three perfectly complementary miRNAs processed from the maternally expressed anti-PEG11 lncRNA [11]. For DLK1, the mechanism of the trans-inhibition remains unknown. The present working model for the callipyge phenomenology is summarized in Additional file 1: Figure S1.

When compared to $+{ }^{\text {Mat }} / C L P G^{\text {Pat }}$ animals, DLK1 levels in CLPG/CLPG animals are more severely reduced at the protein $(\sim 10$-fold $)$ than at the mRNA ( 3 -fold) level $[5,12,13]$. This pattern is compatible with a miRNA-mediated effect that would affect mRNA stability and translation. It made the abundant miRNAs from 
the DLK1-GTL2 domain prime candidate mediators of the observed trans-inhibition. However, bioinformatic analyses did not reveal a striking affinity of any of these miRNAs for DLK1. At best, there was some evidence suggesting that they might have an unusual affinity for the open reading frame (ORF) of $D L K 1$ when assumed to act jointly as a team [10]. As bioinformatic target predictions reputably have limited sensitivity and specificity, we wanted to experimentally evaluate the effect of the miRNAs from the DLK1-GTL2 domain on ovine DLK1.

\section{Results}

Given the prior evidence that (i) the miRNA from the DLK1-GTL2 domain might target the ORF of DLK1 rather than its 3' untranslated region (UTR) [10], and (ii) that their effect might be more pronounced on protein than mRNA concentrations [5], we decided to develop a reporter assay that would (i) express full-length ovine DLK1 transcripts including 5' UTR, ORF and 3' UTR, and (ii) assay protein rather than mRNA levels. We first performed 5' and 3' rapid amplification of cDNA ends (RACE) experiments using RNA from sheep skeletal muscle to accurately map the predominant transcription start and poly-adenylation sites of DLK1 (see Additional file 1: Figure S2). We then assembled a 1,405-bp fragment corresponding to 209-bp of ovine upstream sequence (encompassing the transcription start site mapped 174-bp upstream of the ATG start codon), 954bp of ORF corresponding to the ovine DLK1 C2 isoform appended with a 9-residue human influenza haemagglutinin (HA) carboxyterminal tag, and 242-bp of downstream sequences including an XhoI restriction site (6-bp) and the 236-bp ovine DLK1 3' UTR (ending exactly at the position of the ovine poly-adenylation site) (see Additional file 1: Figure S3A; Additional file 1: Text $\mathrm{S} 1$ ). We selected the $\mathrm{C} 2$ isoform of $D L K 1$ as (i) it is at least 100 times more abundant than the $\mathrm{A}$ isoform in ovine skeletal muscle between -2 and +8 weeks relative to birth, and (ii) the ratio of the transcript levels of the $\mathrm{C} 2$ and $\mathrm{A}$ isoforms is not affected by CLPG genotype (implying that both isoforms encompass the determinants required for the trans-effect) $[5,14]$. The HA tag was introduced because available anti-human DLK1 antibodies did not effectively recognize ovine DLK1 (data not shown). The corresponding fragment was cloned in a slightly modified pcDNA3.1(-) vector, 1-bp downstream of the vector-specific transcriptional start site and 30-bp upstream of the vector-specific bovine growth hormone polyadenylation site. The resulting construct (p3.1M-DLK1-HA) was completely sequenced to confirm its integrity. It was shown, upon transfection in COS1 cells, (i) to generate a transcript of expected size ( 1.4-kb) (Northern blotting), (ii) bounded by the vector-specific transcription start and polyadenylation sites (and hence appended with 1-base of upstream and 30-bases of downstream vector-specific sequences) (5' and 3' RACE), and (iii) producing a protein of expected size recognized by anti-HA antibodies $(\sim 34 \mathrm{kDa}$ after deglycosylation, Western blotting) (see Additional file 1: Figure S3). Within the examined range, DLK1 amounts estimated by phosphorimaging and densitometry increased linearly with loaded quantities $\left(r^{2}=0.98\right)$ (see Additional file 1: Figure S4).

We designed $121 \mathrm{mimic}$ miRNAs, corresponding to one or two isomirs for 108 of the 110 miRNA species from the DLK1-GTL2 domain detected in ovine muscle [10] (see Additional file 2: Table S1 and Methods). In addition, we designed one perfectly complementary antiDLK1 siRNA as positive control and two miRNAs, corresponding to scrambled miR-127 and miR-377, as negative controls (hereafter referred to as siRNA, NC1, and $\mathrm{NC2}$, respectively).

We transfected COS1 cells with a mixture of DLK1expressing p3.1M-DLK1-HA vector, GFP-expressing pcDNA3-GFP vector (to evaluate and correct for variation in transfection efficiency and gel-loaded protein amount), and individual mimic miRNA (Figure 1A). We performed $\geq 4$ independent transfections for each miRNA, and ran $\geq 2$ independent Western blot experiments per transfection, yielding an average of 8.3 usable measures per miRNA. DLK1 amounts, estimated by densitometry, were normalized to GFP amounts as detailed in Methods (see Additional file 1: Figure S5).

As expected (because of its perfect complementarity and predicted cleavage activity), the siRNA positive control exhibited a strong inhibitory effect. It behaved as a complete outlier, reducing the amount of DLK1 3-fold when compared to the average of the two negative controls, and this effect was highly significant $\left(\mathrm{p}=2 \times 10^{-16}\right)$ (Figure 1B and see Additional file 2: Table S2). Seven of the 121 tested miRNAs significantly $(\mathrm{p} \leq 0.05 / 121=4 \times$ $10^{-4}$ ) altered DLK1 levels when compared to the average of the negative controls. One of these caused a reduction in DLK1 levels (miR-329a-3p, 1.25-fold reduction, $\mathrm{p}=$ $\left.3.8 \times 10^{-4}\right)$, while the remaining six increased amounts of DLK1 $\left(\leq 1.28\right.$-fold increase, $\left.\mathrm{p} \geq 1 \times 10^{-8}\right)$. Overall, the 121 miRNAs and the two negative controls appeared to belong to one population (Figure 1B). Accordingly, when compared to the average of all miRNAs (except for the siRNA), one miRNA significantly decreased DLK1 levels (miR-329a-3p, 1.31-fold reduction, $\mathrm{p}=3.2 \times 10^{-5}$ ), and two significantly increased DLK1 levels (miR-376a-5p, 1.25-fold increase, $\mathrm{p}=5 \times 10^{-7}$; miR-432-3p, 1.18-fold increase, $\mathrm{p}=1.8 \times 10^{-5}$ ). MiR-329b-3p, which differs from miR-329a-3p at only two residues near the 3' end, reduced DLK1 amounts 1.1-fold when compared to the overall average, but this was not significant when accounting for multiple testing $(p=0.047)$. MiR-329a-3p 


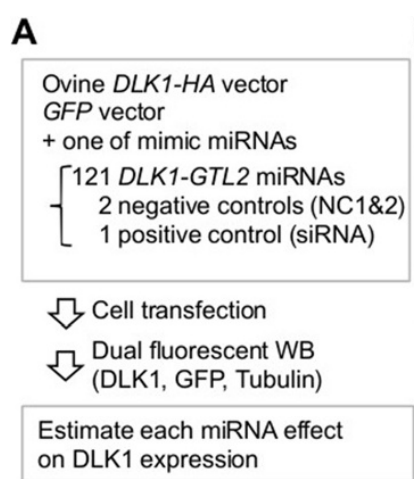

B
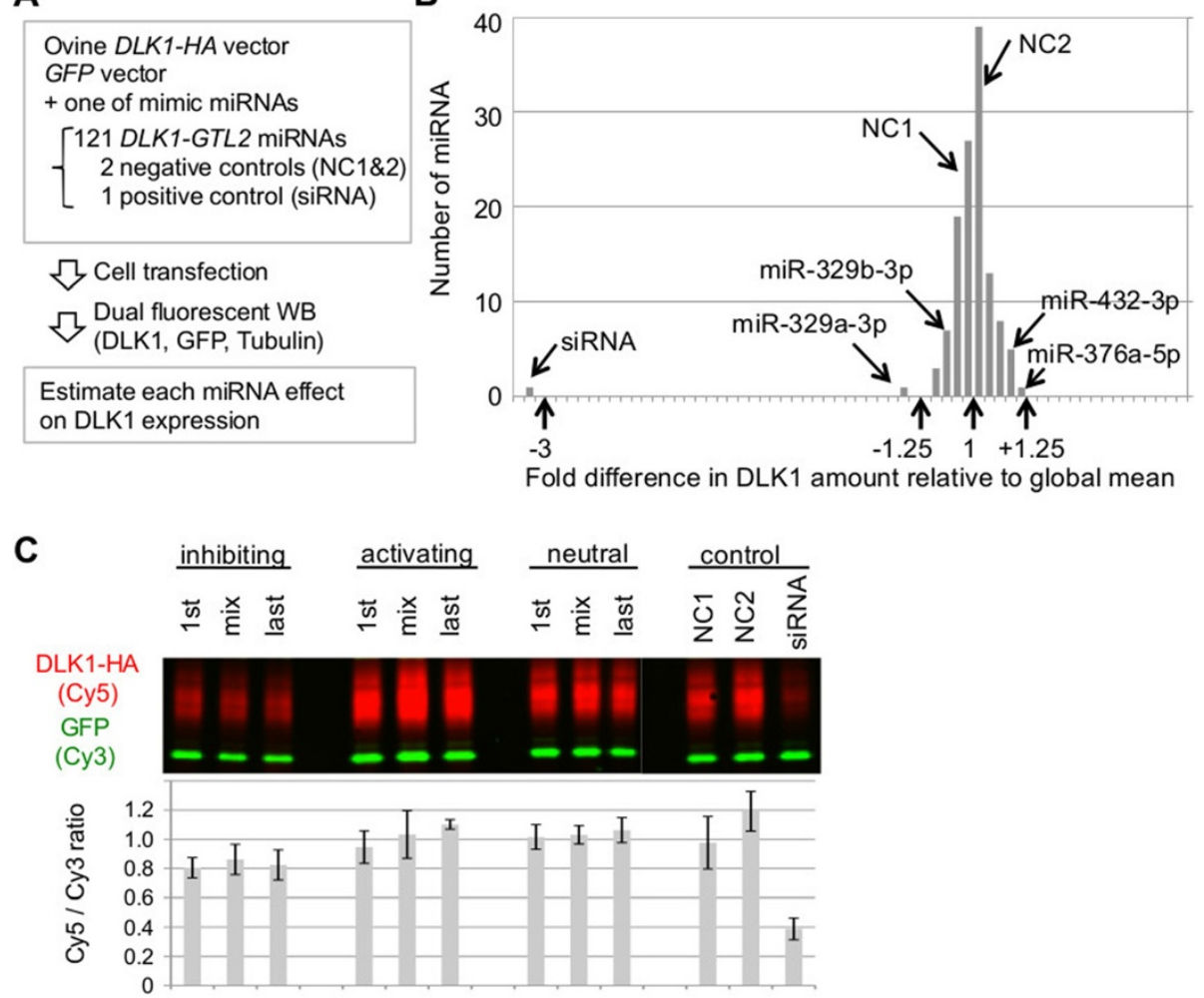

Figure 1 Experimental evaluation of the effect of microRNA from the callipyge locus on DLK1 expression. (A) Overview of experimental procedure of ovine DLK1 reporter assay. We co-transfected COS1 cells with a mixture of ovine full-length (5' UTR, ORF, and 3' UTR) DLK1-expressing vector (p3.1M-DLK1-HA), GFP-expressing vector (pcDNA3-GFP) for normalizing transfection efficiency and gel-loaded protein amount, and one of 121 mimic miRNA candidates from the DLK1-GTL2 imprinted domain, two negative controls (NC1, NC2), and one siRNA targeting the DLK1 ORF as positive control. To facilitate protein quantification, an HA epitope was engineered at the carboxyterminal end of DLK1. Amounts of DLK1, GFP, and endogenous Tubulin were estimated by dual fluorescent Western blot (WB). Effect of each miRNA on DLK1 translation was estimated by normalizing DLK1 with either GFP or Tubulin amounts (corrected DLK1 amount). (B) Distribution of effects of the 121 mimic miRNAs on DLK1 protein amounts. Effects are expressed as fold difference with respect to the overall (minus siRNA) mean. The effects of two negative (NC1, NC2) and one positive (siRNA) controls are shown, as are the effects of the most inhibiting miR-329a-3p, the closely related miR-329b-3p, and the most activating miR-376a-5p and miR-432-3p. GFP was used for normalization. (C) Evaluation of synergistic action of multiple miRNA on DLK1 amount. We selected seven miRNAs consistently showing inhibiting, activating, and neutral effects on DLK1 amount. COS1 cells were co-transfected with the p3.1M-DLK1-HA and PCDNA3-GFP vectors along with either a mixture of the seven miRNAs (mix), or a single miRNA having shown the lowest (1st) or highest (last) corrected DLK1 amount upon transfection in each group. DLK1-HA and GFP protein levels were estimated by dual fluorescent WB analysis (upper panel). Means of corrected DLK1 amounts with standard deviations for four independent experiments are shown (lower panel).

and miR-329b-3p have two 6-mer matches in the ORF and one in the 3' UTR. However, neither of these is evolutionary conserved (see Additional file 1: Figure S6). miR-329a-3p and miR-329b-3p accounted respectively for $0.0014 \%$ and $0.19 \%$ of miRNA molecules derived from the DLK1-GTL2 domain and $0.00043 \%$ and $0.056 \%$ of total miRNA molecules in skeletal muscle of CLPG/ CLPG animals [10]. It is also noteworthy that we did not observe a convincing correlation between effect on DLK1 levels and bioinformatically predicted affinity for $D L K 1$, whether using seed-based scores [15] or Miranda [16], and whether considering the 3' UTR, the ORF, the 5' UTR or the full-length $D L K 1$ transcript (see Additional file 1: Figure S7; Additional file 2: Table S2).
Taken together, these data suggest that none of the tested miRNAs effectively regulates DLK1. Although the effect of miR-329a-3p is significant, we consider the evidence insufficient to claim that it is biologically relevant. Indeed, (i) the effects of the 121 tested miRNAs are approximately normally distributed, encompass the effects of the two negative controls, and are not correlated with predicted affinity for $D L K 1$, (ii) there are more miRNAs that have a significant positive effect than miRNAs that have a negative effect on DLK1 expression (which is unlikely to reflect a genuine biological activity given the known modus operandi of miRNAs), (iii) the effect of miR-329a-3p becomes insignificant when considered jointly with the closely related miR-329b-3p, (iv) the 
expression level of miR-329a-3p in ovine skeletal muscle is extremely low. What is certain is that none of the tested miRNAs is capable - under the utilized conditions - to single-handedly downregulate DLK1 to the extent that is observed in vivo in skeletal muscle of CLPG/CLPG sheep.

It remains possible that the observed trans-inhibition of DLK1 results from the synergistic action of multiple miRNAs. Although we could obviously not test all combinations of miRNAs, we transfected COS1 cells with pools of seven miRNAs chosen (i) amongst the most inhibiting, and (ii) most activating miRNAs. The pools of extremes inhibited, respectively enhanced, DLK1 expression to a degree similar to their constituent miRNAs, but did not reveal convincing evidence for a synergistic effect (Figure $1 \mathrm{C}$ and see Additional file 1: Figure S8).

\section{Discussion}

The aim of this study was to experimentally verify whether any of the miRNAs processed from the ovine DLK1-GTL2 domain might post-transcriptionally downregulate DLK1 to a degree that is commensurate with what is observed in skeletal muscle of CLPG/CLPG sheep. Although one miRNA (miR-329a-3p) yielded an effect deemed significant even when accounting for multiple testing, it was not considered to be relevant with regards to the trans-inhibition of DLK1 in CLPG/CLPG animals for the reasons given above. Of note, when performing ANOVA analysis the "miRNA effect" (not including the three controls) was highly significant $\left(\mathrm{p}<2 \times 10^{-16}\right)$. Thus, the effects of at least some miRNAs are repeatable despite being modest. This could reflect subtle effects on DLK1, on GFP, or on the cellular machinery.

Negative results are in essence uncomfortable to interpret. They may be false negatives because the established reporter assay misses an essential component that is required for the responsible miRNAs to exert their effect. MiRNA-mediated regulation of DLK1 may require auxiliary trans-acting factors not expressed in COS1 cells. The expressed transcripts corresponded for $>93 \%$ to the ovine DLK1 C2 mRNA, yet included 36-bases of extra upstream, 33-bases of extra internal (HA tag), and 30bases of extra downstream sequence, and these may have affected miRNA-target interactions. To discriminate between a miRNA-dependent versus independent mechanism, we have conducted immuno-precipitation experiments using antibodies directed against the Argonaut components of the RISC [17], hoping to show an increase in DLK1 co-immunoprecipitation in skeletal muscle of CLPG/CLPG when compared to $+{ }^{M a t} / C L P G^{\text {Pat }}$ animals, but have so far failed to obtain convincing results (data not shown).
Alternatively, our results may be true negatives and indicate that the trans-inhibition of DLK1 observed in vivo is not a post-transcriptional regulation mediated directly by miRNAs processed from the DLK1-GTL2 domain. It is worthwhile noting in this regard that, while the larger effect on DLK1 protein than on DLK1 transcripts is reminiscent of early reports of the modus operandi of the archetypical animal miRNA lin- 4 on its $l i n-28$ and lin-14 targets in C. elegans, lin-4's mode of action has since been revisited and shown to be more in line with the canonical one in which mRNA destabilization accounts for the major component of repression $[18,19]$. Moreover, the $\sim 3$-fold and $\sim 10$-fold down-regulation of DLK1 observed respectively at the mRNA and protein levels in vivo would be rather extreme for miRNAdependent effects $[20,21]$. Alternative hypotheses include a direct post-transcriptional effect of one or several of the maternally expressed lncRNAs, or an indirect posttranscriptional effect of anyone of the ncRNAs from the domain. As for most lncRNAs, little is known about the function of the maternally expressed GTL2, RIAN and $M I R G$ genes. It has been suggested that GTL2 recruits Polycomb Repressive Complex 2 (PRC2) to downregulate the reciprocally imprinted $D L K 1$ gene in cis [22]. Preliminary results of the co-transfection of COS1 cells with $\mathrm{p} 3.1 \mathrm{M}-D L K 1-\mathrm{HA}$ with a vector expressing the predominant isoform of ovine GTL2 (corresponding to human GTL2 variant 1, NR_002766.2) does not support a direct inhibitory trans-effect of GTL2 on DLK1 expression, on the contrary (see Additional file 1: Figure S9). The hypothesis of an indirect effect posits that the maternally expressed ncRNAs affect targets that do not originate from the DLK1-GTL2 locus, and that these in turn affect DLK1 expression. Such indirect effects may manifest themselves by changes in transcript levels that could be studied by comparing the transcriptome (including small RNAs) of $C L P G^{\text {Mat }} /+{ }^{\text {Pat }}$ with that of $+/+$ animals, and efforts towards that goal are in progress. Along similar lines, Bidwell et al. [23] recently showed that muscle of CLPG/CLPG animals exhibited lower expression of a group of key regulatory genes for muscle development (e.g. ZFP106, RPS6KA3, MEF2A), that might render them less responsive to the hypertrophic stimuli of the paternal allele-specific genes.

The apparently stronger effect on $D L K 1$ protein $(\sim 10$ fold) than on DLK1 transcript ( $\sim 3$-fold) level suggests a post-transcriptional mechanism. This hypothesis received considerable support from the demonstration of the RISC-mediated cleavage of PEG11 by miRNAs processed from anti-PEG11 [11]. However, we cannot totally exclude a transcriptional mechanism. It may be worthwhile, in this regard, to perform ribosome profiling [24] experiments to verify whether the predicted translational inhibition of DLK1 transcripts in CLPG/CLPG animals 
when compared to $+{ }^{\mathrm{Mat}} / C L P G^{\mathrm{Pat}}$ animals is indeed observed. Lack of such effect would force us to reevaluate scenarios involving transcriptional regulation.

More work will be needed to gain a full understanding of the molecular mechanisms underlying the trans-inhibition of $D L K 1$, which is key element of polar overdominance at the CLPG locus.

\section{Conclusions}

Contrary to that of PEG11, the trans-inhibition of DLK1 that is observed in CLPG/CLPG sheep and lies at the heart of polar overdominance, may not depend on posttranscriptional regulation by miRNAs from the DLK1GTL2 domain.

\section{Methods}

\section{Ethical statement}

All animal procedures were carried out in strict accordance with the recommendations in the Guide for the Care and Use of Laboratory Animals of Utah State University and approved by the Animal Ethics Committees of Utah State University (IACUC \#386).

\section{RACE}

The 5' and 3' ends of DLK1 transcripts were determined using the GeneRacer Kit (Invitrogen, Carlsbad, CA) according to the manufacturer's instructions. Briefly, total RNA was isolated either from skeletal muscle (longissimus dorsi) of 2-week-old sheep of the four possible CLPG genotypes (+/+, $+{ }^{\text {Mat }} / C L P G^{\text {Pat }}, C L P G^{\text {Mat }} /+{ }^{\text {Pat }}$, CLPG/CLPG), or from COS1 cells $24 \mathrm{hrs}$ after transfection with the ovine $D L K 1$ expressing p3.1M-DLK1-HA vector. For 5' RACE, $3 \mu \mathrm{g}$ of total RNA was used for ligation of the GeneRacer RNA Oligo to the 5' end of RNA with a 5' cap structure. RNA was then reverse transcribed using SuperScript III reverse transcriptase using random hexamers. For 3' RACE, cDNA was synthesized using the GeneRacer Oligo dT primer. These cDNAs were then amplified with the GeneRacer 5' and DLK1specific 5' GSP-A primers for 5' RACE, and the GeneRacer 3' and DLK1-specific 3' GSP-S primers for 3' RACE, respectively. Nested PCR amplifications were performed using diluted PCR products with the GeneRacer 5' nested and DLK1-specific 5' NGSP-A primers for 5' RACE, and the GeneRacer 3' nested and DLK1-specific 3' NGSP-S primers for 3' RACE, respectively. For both 5' RACE PCRs, we used the Expand Long Template PCR System (Roche Diagnostics, Basel, Switzerland) with PCR conditions: $94^{\circ} \mathrm{C}$ for $2 \mathrm{~min} ; 10$ cycles of $94^{\circ} \mathrm{C}$ for 10 sec, $62^{\circ} \mathrm{C}$ for $30 \mathrm{sec}$ and $68^{\circ} \mathrm{C}$ for $1 \mathrm{~min} ; 22$ cycles of $94^{\circ} \mathrm{C}$ for $15 \mathrm{sec}, 62^{\circ} \mathrm{C}$ for $30 \mathrm{sec}$ and $68^{\circ} \mathrm{C}$ for $1 \mathrm{~min}$; and $68^{\circ} \mathrm{C}$ for $7 \mathrm{~min}$. We used the Phusion DNA polymerase (Finnzymes, Espoo, Finland) for the 3' RACE PCRs using touchdown PCR condition: $98^{\circ} \mathrm{C}$ for $30 \mathrm{sec} ; 8$ cycles of $98^{\circ} \mathrm{C}$ for $15 \mathrm{sec}, 66^{\circ} \mathrm{C}$ for $20 \mathrm{sec}$ and $72^{\circ} \mathrm{C}$ for $40 \mathrm{sec} ; 20$ cycles of $98^{\circ} \mathrm{C}$ for $15 \mathrm{sec}, 62^{\circ} \mathrm{C}$ for $20 \mathrm{sec}$ and $72^{\circ} \mathrm{C}$ for $40 \mathrm{sec}$; and $72^{\circ} \mathrm{C}$ for $3 \mathrm{~min}$. The nested PCR products were analyzed by agarose gel electrophoresis, purified from the gel, and sequenced either directly or after subcloning in the pCR2.1 TA cloning vector (Invitrogen) to determine the 5' and 3' ends of the DLK1 transcripts. All primer sequences are listed in Additional file 2: Table S3.

\section{Generation of the p3.1M-DLK1-HA DLK1 expression vector} The ovine DLK1 ORF (C2 isoform) was PCR-amplified using ovine skeletal muscle cDNA with primers p3.1-CS and p3.1-CA containing $\mathrm{XbaI}$ and $\mathrm{XhoI}$ sites at their 5' ends, respectively. The reverse primer (p3.1-CA) included a HA tag sequence (AGCGTAGTCTGGG ACGTCGTATGGGTA) to add the HA epitope at the carboxyterminus of ovine DLK1 protein. The XbaI/XhoI double-digested PCR fragment was cloned into the XbaI/XhoI sites of the pcDNA3.1(-) mammalian expression vector (Invitrogen) to create an intermediate vector p3.1-DLK1-CDS-HA. The 5' UTR and a part of the coding sequence of ovine $D L K 1$ was amplified from genomic DNA using the Expand Long Template PCR system with primers $\mathrm{p} 3.1-5 \mathrm{~S}$ containing an $\mathrm{XbaI}$ site at the 5 ' end and p3.1-5A encompassing an endogenous NcoI site in the middle of the primer. The ovine DLK1 3' UTR was PCR-amplified using genomic DNA with primers, p3.1-3S and p3.1-3A containing XhoI and EcoRI sites at their 5' ends, respectively. The two resultant PCR fragments were double-digested with $\mathrm{XbaI} / \mathrm{NcoI}$ and $X h o I / E c o R I$, respectively, and inserted sequentially into the p3.1-DLK1-CDS-HA vector at the corresponding sites to generate the $\mathrm{p} 3.1-D L K 1-\mathrm{FL}-\mathrm{HA}$ vector. To minimize vector sequence in the transcripts (more specifically the multiple cloning region between the transcription start and end sites of the vector), we modified the original pcDNA3.1(-) vector (referred to as p3.1M). We introduced an $X b a \mathrm{I}$ recognition site adjacent to the putative transcriptional start site and an EcoRI site upstream of the bovine growth hormone polyadenylation signal of the vector using the Quickchange lightening site-directed mutagenesis kit (Stratagene, La Jolla, CA) with oligonucleotides pc3.1-XbaI-intro-U/D and pc3.1EcoRI-intro-U/D, respectively. The insert of the p3.1DLK1-FL-HA (containing the HA tagged full-length of ovine DLK1) was excised by an XbaI/EcoRI doubledigestion and transferred to the modified $\mathrm{p} 3.1 \mathrm{M}$ vector at the corresponding sites to generate the final p3.1MDLK1-HA vector. Orientation and integrity of the 1,405bp insert was confirmed by sequencing using the BigDye Terminator v3.1 Cycle Sequencing kits and the 3730 DNA Analyzer (Applied Biosystems, Foster City, CA) in three sequencing reactions using primers, Seq-S, Seq-A, 
and 3' NGSP-S. Primer information is available in Additional file 2: Table S3.

\section{Mimic miRNAs - choice of the system}

To co-express miRNAs from the DLK1-GTL2 domain with the vector expressing $D L K 1$, we initially elected to clone the corresponding miRNA precursors in expression vectors and co-transfect them. The hope was that one vector would allow for the expression of multiple miRNA species and isomirs (one miRNA precursor can give rise to two miRNA "species" (from the $5 p$ and $3 p$ arms, respectively), that can each come in multiple forms or "isomirs"), and that this would optimally mimic the condition prevailing in vivo at minimum cost. We realized, however, that the expression levels of the different miRNA species varied enormously, without necessarily mimicking the in vivo ratios [10], at concentrations that would often be $<1 \%$ of endogenous let $7 d$ levels (data not shown). As this would make comparisons between miRNAs nearly impossible, we opted for the use of synthetic "mimic" miRNAs.

\section{Mimic miRNAs - molecular design}

We evaluated the efficacy of different designs of mimic miRNAs using the luciferase assay described in Takeda et al. [17] (see Additional file 1: Figure S10). It is based on the pRL-4xTex and pRL-4xRom vectors, that each contains four tandem repeats respectively with and without miR-1 target sites. We compared four types of mimic miRNAs: (i) an unmodified RNA duplex corresponding to the endogenous mature and star ovine miR1 miRNAs (hence with bulge, wobble base pairs and mismatch), (ii) an unmodified RNA duplex corresponding to the mature ovine miR-1 and its perfect reverse complement, with 2-nt 3' overhangs at both ends (UU for the passenger strand), (iii) the same as in (ii) but with modified 5' ends aimed at promoting the incorporation of the guide strand into the RISC complex: 5' $\mathrm{P}$ (phosphate) for the mature miRNA strand and/or $5^{\prime} \mathrm{NH}_{2}$ for the passenger strand, and (iv) the proprietary miR-1 miRNA Precursor purchased from Ambion. While type (i) (i.e. a copy of the endogenous double-stranded miRNA) did not have any effect, types (ii), (iii) and (iv) worked equally well. We opted for type (ii) with unmodified mature strand and $5^{\prime} \mathrm{NH}_{2}$ modified passenger strand. The 3 ' overhangs for all passenger strands corresponded to UU dinucleotides. Corresponding oligonucleotides were purchased from GenePharma (Shanghai, China).

\section{Mimic miRNAs - sequence determination}

To select representative mimic miRNA sequences for all miRNAs from the DLK1-GTL2 domain, we simultaneously utilized (i) previously generated RNA-Seq information that was used to establish a catalogue of miRNAs expressed in ovine skeletal muscle with special emphasis on the DLK1-GTL2 domain [10], (ii) miRNA characteristics inferred from meta-analyses of mouse and Drosophila melanogaster small RNAs [25,26], and (iii) information of human, mouse, and bovine orthologs from miRBase [27]. In most cases, the selected sequences corresponded to the most abundant isomirs in the ovine RNA-Seq data, matching the miRBase registered sequences, and formed a canonical mature and star miRNA duplex with 3' overhangs. To those, we added (i) "star" sequences that were not registered in the miRBase but were observed in our RNA-Seq data, and (ii) alternative isomir with 5' heterogeneity that represented more than one third of the reads of a corresponding miRBaseregistered miRNA or with more than 200 reads in any of the RNA-Seq libraries. A-to-I editing [10] or untemplated nucleotide addition (data not shown) were observed in some of miRNAs from the DLK1-GTL2 domain. This was accounted for by synthesizing isomirs with mixed bases at the corresponding sites. The complete list of 121 tested mimic miRNAs is provided in Additional file 2: Table S1. MiRNAs derived from the $5 p$ and $3 p$ arms of a miRNA hairpin precursor are suffixed with $-5 p$ and $-3 p$, respectively, irrespective of their relative expression levels.

To design negative control miRNA, we first shuffled sequences of five miRNAs (oar-miR-433-3p, 127-3p, 3957-3p, 377-3p, and 376c-3p) ten times each. We then counted the number of 8 -mer, 7 mer-A1, 7mer-m8, and 6-mer seed matches [15] of these shuffled sequences to the full-length ovine $D L K 1 \mathrm{C} 2$ isoform. In addition, to minimize off-target effects on unintended endogenous RNAs, we predicted the number of genome wide conserved miRNA target sites across most mammals using the TargetScan Custom software [28,29]. Two of the shuffled sequences that showed neither 6-, 7-nor 8-mer seed matches to the ovine $D L K 1$ and the lowest number of predicted genome-wide conserved target sites (seven and one conserved targets, respectively) were selected as negative controls (designated as Scr-oar-miR-127-3p (NC1) and Scr-oar-miR-377-3p (NC2)). In addition, we purchased one siRNA targeting the ovine DLK1 ORF by using the BLOCK-iT RNAi Designer (Invitrogen), to be used as positive control.

\section{Cell culture and transient transfection}

COS1 cells were cultured in Dulbecco's modified Eagle's medium supplemented with $10 \%$ fetal bovine serum and $0.1 \mathrm{mM}$ non-essential amino acids, 100 units $/ \mathrm{ml}$ penicillin and $100 \mathrm{mg} / \mathrm{ml}$ streptomycin at $37^{\circ} \mathrm{C}$ (GIBCO, Renfrewshire, UK). One day before transfection, $0.5 \times$ $10^{5}$ cells were seeded in a 12 -well plate in $800 \mu \mathrm{l}$ of growth medium without antibiotics. We then co- 
transfected the cells with $200 \mu \mathrm{l}$ of Opti-MEM medium (GIBCO) containing $200 \mathrm{ng}$ of the $\mathrm{p} 3.1 \mathrm{M}-D L K 1-\mathrm{HA}$ vector, $400 \mathrm{ng}$ of pcDNA3-GFP vector (Plasmid ID: 13031 obtained from Addgene, Cambridge, MA) along with 40 pmols of synthetic mimic miRNA (GenePharma)(final concentration $40 \mathrm{nM}$ ) or 5 pmols of siRNA (Invitrogen) using $4 \mu \mathrm{l}$ of Lipofectamin 2000 (Invitrogen) according to the manufacturer's instructions.

\section{Dual fluorescence Western blotting}

Total protein was extracted from COS1 cells $24 \mathrm{hrs}$ after transfection using a modified RIPA buffer containing 50 $\mathrm{mM}$ Tris- $\mathrm{HCl}$ (pH 7.6), $150 \mathrm{mM} \mathrm{NaCl}, 1 \%$ Igepal CA630, $0.25 \%$ sodium deoxycholate, $1 \mathrm{mM}$ EDTA and the complete protease inhibitor cocktail (Roche). The lysate was centrifuged at $12,000 \times \mathrm{g}$ for $15 \mathrm{~min}$ at $4^{\circ} \mathrm{C}$ and protein concentration of the supernatant was determined using the micro BCA Protein Assay Kit (Pierce, Rockford, IL) according to the manufacturer's instructions. Eight $\mu$ g of protein were boiled for $5 \mathrm{~min}$ in Laemmli buffer (62.5 mM Tris- $\mathrm{HCl}$ (pH 7.6), 2\% sodium dodecyl sulfate (SDS), $10 \%$ glycerol, $0.004 \%$ bromophenol blue, $5 \% \beta$-mercaptoethanol) and loaded on a NuPAGE Novex 4-12\% Bis-Tris Midi gel (Invitrogen). Electrophoresis was performed with the XCell4 SureLock Midi-Cell electrophoresis chamber (Invitrogen) at $200 \mathrm{~V}$ for $30 \mathrm{~min}$ at room temperature in the NuPAGE MOPS SDS Running buffer (Invitrogen). Protein was then transferred to a Hybond-LFP Polyvinylidene difluoride (PVDF) membrane (GE Healthcare, Buckinghamshire, UK) at $200 \mathrm{~mA}$ for $90 \mathrm{~min}$ at $4^{\circ} \mathrm{C}$ using the NuPAGE transfer buffer (Invitrogen) in a Criterion Blotter (BioRad, Hercules, $\mathrm{CA})$. The membrane was blocked with $10 \%$ non-fat dry milk in PBST buffer $\left(1 \mathrm{mM} \mathrm{KH} \mathrm{PO}_{4}, 10 \mathrm{mM} \mathrm{Na} 2 \mathrm{HPO}_{4}\right.$, $137 \mathrm{mM} \mathrm{NaCl}, 2.7 \mathrm{mM} \mathrm{KCl}, 0.1 \%$ Tween 20) either at room temperature for $1 \mathrm{hr}$ or at $4^{\circ} \mathrm{C}$ overnight. The membrane was incubated sequentially with a series of antibodies diluted in the blocking buffer at room temperature for $1 \mathrm{hr}$. We used a rabbit polyclonal antiHA tag antibody (1:4000 dilution) (ab9110, Abcam, Cambridge, UK), an ECL Plex anti-rabbit IgG antibody conjugated with Cy5 dye (1:2500) (PA45011, GE Healthcare), a mouse monoclonal anti-GFP antibody (1:1000) (clone JL-8, Clontech Laboratories, Mountain View, CA), a mouse monoclonal anti- $\alpha$-Tubulin antibody (1:8000) (T6074, Sigma, St. Louis, MO), and an ECL Plex anti-mouse IgG antibody conjugated with Cy3 dye (1:4000) (PA43010, GE Healthcare). Membranes were washed three times for $10 \mathrm{~min}$ in PBST buffer with gentle shaking between incubations. The membrane was then dried at $37^{\circ} \mathrm{C}$ for $1 \mathrm{hr}$, followed by scanning with the Typhoon 9400 scanner (GE healthcare). Band intensities corresponding to HA-tagged DLK1, GFP, and Tubulin were quantified using the ImageQuant TL software (GE healthcare). For normalization, the band intensities were first divided by the corresponding membrane-specific averages (not including siRNA), yielding "relative" DLK1, GFP, Tubulin amounts. The relative DLK1 amounts were then divided by either the corresponding relative GFP or Tubulin amounts, yielding "corrected" DLK1 amounts. We performed more than four independent transfections of cells for each mimic miRNA, and ran more than two independent Western blot experiments per transfection. MiRNA effect on the DLK1 expression was estimated by comparing the mean of the corrected DLK1 amounts for a given miRNA either to the global mean (not including siRNA data) or to the average of $\mathrm{NC} 1$ and $\mathrm{NC} 2$ data.

\section{Deglycosylation}

As DLK1 has been reported to be $\mathrm{N}$ - and $\mathrm{O}$-glycosylated [30], we carried out Western blot analysis using proteins treated with the Protein Deglycosylation mix (New England Biolabs, Ipswich, MA) according to the manufacturer's instructions. Briefly, COS1 cells transfected with the p3.1M-DLK1-HA vector were harvested in the Phosphate Buffered Saline (GIBCO) supplied with 1\% Igepal CA-630 and the complete protease inhibitor cocktail. Twenty $\mu \mathrm{g}$ of protein were denatured at $99^{\circ} \mathrm{C}$ for $10 \mathrm{~min}$ and treated at $37^{\circ} \mathrm{C}$ for $4 \mathrm{hrs}$ with a mixture of deglycosylation enzymes that were expected to remove all $N$-linked and simple $O$-linked glycans, as well as some complex $O$-linked glycans. Western blot was performed as described above.

\section{Northern blot analysis}

Total RNA was isolated from COS1 cells $24 \mathrm{hrs}$ after transfection with the p3.1M-DLK1-HA vector using the Trizol reagent (Invitrogen) according to the manufacturer's protocol. Ten $\mu \mathrm{g}$ of total RNA was denatured at $50^{\circ} \mathrm{C}$ for $10 \mathrm{~min}$ in $20 \mu \mathrm{l}$ of $1 \times$ MOPS buffer $(20 \mathrm{mM} \mathrm{3-}$ (N-morpholino) propanesulfonic acid, $5 \mathrm{mM}$ sodium acetate, $1 \mathrm{mM}$ EDTA, $1 \mathrm{mM}$ EGTA, pH 7.0) supplied with $2.4 \mathrm{M}$ formaldehyde and $50 \%$ formamide. The RNA was mixed with loading buffer (final 5\% glycerol, 0.025\% bromophenol blue, $0.025 \%$ xylene cyanol) and subjected to electrophoresis on a $1 \%$ agarose gel containing 1.75 $\mathrm{M}$ formaldehyde in $1 \times$ MOPS buffer at $5 \mathrm{~V} / \mathrm{cm}$ for 3.5 hrs. After electrophoresis, the RNA was transferred onto a Hybond- $\mathrm{N}^{+}$nylon membrane (GE Healthcare) with $10 \times$ SSC buffer $(150 \mathrm{mM}$ sodium citrate, $1.5 \mathrm{M} \mathrm{NaCl}$, $\mathrm{pH}$ 7.0) and UV cross-linked essentially as described in Sambrook et al. [31]. A DNA fragment containing the ovine DLK1 ORF region (857-bp) was gel-purified after digesting the plasmid p3.1M-DLK1-HA with NcoI and $B g l \mathrm{II}$ and labeled with $\left[\alpha^{32} \mathrm{P}\right] \mathrm{dCTP}$ (GE Healthcare) using the Prime-It II random primer labeling kit (Stratagene). The membrane was pre-hybridized with the 
ULTRAhyb buffer (Ambion) at $42^{\circ} \mathrm{C}$ for 2 hrs and then hybridized with $1 \times 10^{6} \mathrm{cpm} / \mathrm{ml}$ of the ${ }^{32}$ P-labeled probe at $45^{\circ} \mathrm{C}$ overnight. Membranes were washed twice at $55^{\circ} \mathrm{C}$ for $20 \mathrm{~min}$ in $2 \times \mathrm{SSC}$ and $0.1 \%$ SDS, then twice at $55^{\circ} \mathrm{C}$ for $30 \mathrm{~min}$ in $0.1 \times$ SSC and 0.1\% SDS. Membranes were exposed to the Hyperfilm ECL film (GE Healthcare) for 3 hrs.

\section{Generation of ovine GTL2 expression vector}

Ovine GTL2 sequence (see Additional file 1: Text S2) was PCR-amplified from cDNA synthesized with random hexamers from longissimus dorsi skeletal muscle RNA of an 8-week-old CLPG/CLPG animal using the Phusion DNA polymerase with primers, ovGTL2-FL-S1 and ovGTL2-FL-A1 (see Additional file 2: Table S3). The resultant PCR product was sub-cloned into the pCRII-blunt-TOPO vector (Invitrogen) and subjected to DNA sequencing for confirmation. The insert was excised with $E c o R I$ and ligated into the corresponding site of the pcDNA3.1(-) vector to produce the p3.1-GTL2 and p3.1-antiGTL2 vectors that express ovine GTL2 in sense and antisense orientations, respectively. Integrity of the insert was confirmed by sequencing.

\section{In silico affinity score of miRNA to DLK1}

Affinity scores of each miRNA for ovine $D L K 1$ transcript expressed from the p3.1M-DLK1-HA were computed using Miranda [16] and a seed-based score [15]. Miranda scores were obtained by running the Miranda v3.3a software using the miRNA sequences (see Additional file 2: Table S1) and the ovine DLK1 5' UTR, ORF, 3' UTR, and full-length sequences (see Additional file 1: Text S1). For the Grimson seed match score, we simply counted the number of sites complementary to miRNA seed sequences (8mer, 7mer-A1, 7mer-m8, 6mer, and 6mer-offset seed matches [15]) on the DLK1 sequences. Pearson correlation coefficients between the prediction scores and the corrected DLK1 amounts (DLK1/GFP) (without controls) and its significant levels were calculated using standard procedures.

\section{Additional files}

Additional file 1: Figure S1. Working model for polar overdominance at the ovine callipyge locus [11]. Figure S2: Characterization of the $5^{\prime}$ and $3^{\prime}$ ends of ovine DLK1 transcripts in ovine skeletal muscle. Figure S3: Construction and characterization of the p3.1M-DLK1-HA vector expressing full length ovine DLK1 [30]. Figure S4: Quantitativeness of Western blot analysis of ovine DLK1 protein. Figure S5: Dual fluorescent Western blot to measure DLK1 amount. Figure S6: Conservation around oar-miR-329a-3p 6-mer seed matches on DLK1 [15,32]. Figure S7: Weak correlation between miRNA-target affinity score and DLK1 $[15,16]$. Figure S8: MiRNAs used for a multiple-miRNA transfection test. Figure S9: Effect of GTL2 IncRNA expression on DLK1 amount. Figure S10: Functionality of synthetic mimic miRNAs [17]. Text S1: RNA sequence transcribed from the p3.1M-DLK1-HA expression vector. Text S2: Ovine GTL2 sequence in the p3.1-GTL2 expression vector.

Additional file 2: Table S1. Mimic miRNA sequence information [10,33]. Table S2: Effect of each mimic miRNA on DLK1 expression and its correlation with predicted affinity scores $[15,16]$. Table S3: Oligonucleotides used in this study.

\section{Abbreviations}

CLPG: Callipyge; DLK1: Delta-like 1 homolog (Drosophila); PEG11: Paternally expressed gene 11; RTL1: Retrotransposon-like 1; GTL2: Gene trap locus 2; RIAN: RNA imprinted and accumulated in nucleus; MIRG: MicroRNA containing gene; miRNA: microRNA; ncRNA: non-coding RNA; IncRNA: long non-coding RNA; snoRNA: small nucleolar RNA; dsRNA: double stranded RNA; ORF: Open reading frame; UTR: Untranslated region; PRC2: Polycomb repressive complex 2; RACE: Rapid amplification of CDNA ends; HA: Haemagglutinin; RISC: RNA-induced silencing complex; moR: miRNAoffset RNA.

\section{Competing interests}

The authors declare that they have no competing interests.

\section{Authors' contributions}

$H C, X X, C C, M G$ and $H T$ contributed to the conception and design of the study, and analysis and interpretation of data. TH and NC provided sheep samples. $\mathrm{HC}$ performed experiments. HC, MG, and $\mathrm{HT}$ drafted the manuscript. CC, TH and NC helped to draft the manuscript. All authors read and approved the final manuscript.

\section{Acknowledgments}

We would like to thank Dr. Zissimos Mourelatos (University of Pennsylvania) and Dr. Yukihide Tomari (University of Tokyo) for advice in mimic miRNA design, and Ms Xin Zhang, Dr. Ming Fang and Dr. Kateryna Shostak (University of Liège) for their kind help to HC. We also thank the GIGA sequencing facility (University of Liège) for their support. This work was funded by the FNRS (project 2.4544.11), by the Communauté Française de Belgique (ARC Mirage), by China state-sponsored post-graduate study abroad program (to $\mathrm{HC}$ ), and by BELSPO (postdoctoral fellowship to XX).

\section{Author details}

${ }^{1}$ Unit of Animal Genomics, GIGA-R \& Faculty of Veterinary Medicine, University of Liège (B34), 1 Avenue de l'Hôpital, 4000 Liège, Belgium. ${ }^{2}$ Key Lab of Agricultural Animal Genetics, Breeding and Reproduction of Ministry of Education, College of Animal Science \& Technology, Huazhong Agricultural University, Wuhan 430070, P. R. China. ${ }^{3}$ Department of Animal, Dairy and Veterinary Sciences, Utah State University, Logan, UT 84322, USA.

Received: 15 June 2014 Accepted: 16 October 2014 Published: 30 October 2014

\section{References}

1. Cockett NE, Jackson SP, Shay TL, Farnir F, Berghmans S, Snowder GD, Nielsen DM, Georges M: Polar overdominance at the ovine callipyge locus. Science 1996, 273:236-238.

2. Lawson HA, Cheverud JM, Wolf JB: Genomic imprinting and parent-of-origin effects on complex traits. Nat Rev Genet 2013, 14:609-617.

3. Charlier C, Segers K, Karim L, Shay T, Gyapay G, Cockett N, Georges M: The callipyge mutation enhances the expression of coregulated imprinted genes in cis without affecting their imprinting status. Nat Genet 2001, 27:367-369.

4. Freking BA, Murphy SK, Wylie AA, Rhodes SJ, Keele JW, Leymaster KA, Jirtle $R L$, Smith TP: Identification of the single base change causing the callipyge muscle hypertrophy phenotype, the only known example of polar overdominance in mammals. Genome Res 2002, 12:1496-1506.

5. Davis $\mathrm{E}$, Jensen $\mathrm{CH}$, Schroder HD, Farnir F, Shay-Hadfield T, Kliem A, Cockett N, Georges M, Charlier C: Ectopic expression of DLK1 protein in skeletal muscle of padumnal heterozygotes causes the callipyge phenotype. Curr Biol 2004, 14:1858-1862.

6. Byrne K, Colgrave ML, Vuocolo T, Pearson R, Bidwell CA, Cockett NE, Lynn DJ, Fleming-Waddell JN, Tellam RL: The imprinted retrotransposon-like 
gene PEG11 (RTL1) is expressed as a full-length protein in skeletal muscle from Callipyge sheep. PLoS One 2010, 5:e8638.

7. Georges M, Charlier C, Cockett N: The callipyge locus: evidence for the trans interaction of reciprocally imprinted genes. Trends Genet 2003, 19:248-252

8. Charlier C, Segers K, Wagenaar D, Karim L, Berghmans S, Jaillon O, Shay T, Weissenbach J, Cockett N, Gyapay G, Georges M: Human-ovine comparative sequencing of a 250-kb imprinted domain encompassing the callipyge (clpg) locus and identification of six imprinted transcripts: DLK1, DAT, GTL2, PEG11, antiPEG11, and MEG8. Genome Res 2001, 11:850-862.

9. Edwards CA, Mungall AJ, Matthews L, Ryder E, Gray DJ, Pask AJ, Shaw G, Graves JA, Rogers J, Savoir consortium, Dunham I, Renfree MB, Ferguson-Smith AC: The evolution of the DLK1-DIO3 imprinted domain in mammals. PLOS Biol 2008, 6:e135.

10. Caiment F, Charlier C, Hadfield T, Cockett N, Georges M, Baurain D: Assessing the effect of the CLPG mutation on the microRNA catalog of skeletal muscle using high-throughput sequencing. Genome Res 2010, 20:1651-1662.

11. Davis E, Caiment F, Tordoir X, Cavaille J, Ferguson-Smith A, Cockett N, Georges M, Charlier C: RNAi-mediated allelic trans-interaction at the imprinted Rt11/Peg11 locus. Curr Biol 2005, 15:743-749.

12. Murphy SK, Freking BA, Smith TP, Leymaster K, Nolan CM, Wylie AA, Evans $H K$, Jirtle RL: Abnormal postnatal maintenance of elevated DLK1 transcript levels in callipyge sheep. Mamm Genome 2005, 16:171-183.

13. White JD, Vuocolo T, McDonagh M, Grounds MD, Harper GS, Cockett NE, Tellam R: Analysis of the callipyge phenotype through skeletal muscle development; association of Dlk1 with muscle precursor cells. Differentiation 2008, 76:283-298.

14. Vuocolo T, Cockett NE, Tellam RL: Expression of imprinted genes surrounding the callipyge mutation in ovine skeletal muscle. Aust J Exp Agric 2005, 45:879-892.

15. Grimson A, Farh KK, Johnston WK, Garrett-Engele P, Lim LP, Bartel DP: MicroRNA targeting specificity in mammals: determinants beyond seed pairing. Mol Cell 2007, 27:91-105.

16. John B, Enright AJ, Aravin A, Tuschl T, Sander C, Marks DS: Human MicroRNA targets. PLoS Biol 2004, 2:e363.

17. Takeda H, Charlier C, Farnir F, Georges M: Demonstrating polymorphic miRNA-mediated gene regulation in vivo: application to the $\mathrm{g}+$ 6223G- > A mutation of Texel sheep. RNA 2010, 16:1854-1863.

18. Bagga S, Bracht J, Hunter S, Massirer K, Holtz J, Eachus R, Pasquinelli AE: Regulation by let-7 and lin-4 miRNAs results in target mRNA degradation. Cell 2005, 122:553-563.

19. Guo H, Ingolia NT, Weissman JS, Bartel DP: Mammalian microRNAs predominantly act to decrease target mRNA levels. Nature 2010 466:835-840.

20. Baek D, Villen J, Shin C, Camargo FD, Gygi SP, Bartel DP: The impact of microRNAs on protein output. Nature 2008, 455:64-71.

21. Selbach M, Schwanhausser B, Thierfelder N, Fang Z, Khanin R, Rajewsky N: Widespread changes in protein synthesis induced by microRNAs. Nature 2008, 455:58-63.

22. Zhao J, Ohsumi TK, Kung JT, Ogawa Y, Grau DJ, Sarma K, Song JJ, Kingston RE, Borowsky M, Lee JT: Genome-wide identification of polycombassociated RNAs by RIP-seq. Mol Cell 2010, 40:939-953.

23. Bidwell CA, Waddell JN, Taxis TM, Yu H, Tellam RL, Neary MK, Cockett NE: New insights into polar overdominance in callipyge sheep. Anim Genet 2014, 45(Suppl 1):51-61.

24. Ingolia NT, Ghaemmaghami S, Newman JR, Weissman JS: Genome-wide analysis in vivo of translation with nucleotide resolution using ribosome profiling. Science 2009, 324(5924):218-223.

25. Chiang HR, Schoenfeld LW, Ruby JG, Auyeung VC, Spies N, Baek D, Johnston WK, Russ C, Luo S, Babiarz JE, Blelloch R, Schroth GP, Nusbaum C, Bartel DP: Mammalian microRNAs: experimental evaluation of novel and previously annotated genes. Genes Dev 2010, 24:992-1009.

26. Berezikov E, Robine N, Samsonova A, Westholm JO, Naqvi A, Hung JH, Okamura K, Dai Q, Bortolamiol-Becet D, Martin R, Zhao Y, Zamore PD, Hannon GJ, Marra MA, Weng Z, Perrimon N, Lai EC: Deep annotation of Drosophila melanogaster microRNAs yields insights into their processing modification, and emergence. Genome Res 2011, 21(2):203-215.

27. Griffiths-Jones S: The microRNA Registry. Nucleic Acids Res 2004 32:D109-D111.
28. TargetScan custom [http://www.targetscan.org/vert_50/seedmatch.html]

29. Lewis BP, Burge CB, Bartel DP: Conserved seed pairing, often flanked by adenosines, indicates that thousands of human genes are microRNA targets. Cell 2005, 120:15-20.

30. PhosphoSitePlus (DLK1) [http://www.phosphosite.org/proteinAction.do? id $=21757 \&$ showAllSites=true]

31. Sambrook J, Fritsch EF, Maniatis T: Molecular Cloning: A Laboratory Manual. 1989.

32. Blanchette M, Kent WJ, Riemer C, Elnitski L, Smit AF, Roskin KM, Baertsch R, Rosenbloom K, Clawson H, Green ED, Haussler D, Miller W: Aligning multiple genomic sequences with the threaded blockset aligner. Genome Res 2004, 14:708-715.

33. Shi W, Hendrix D, Levine M, Haley B: A distinct class of small RNAs arises from pre-miRNA-proximal regions in a simple chordate. Nat Struct Mol Biol 2009, 16:183-189.

doi:10.1186/1471-2164-15-944

Cite this article as: Cheng et al: Experimental evaluation does not reveal a direct effect of microRNA from the callipyge locus on DLK1 expression. BMC Genomics 2014 15:944.

\section{Submit your next manuscript to BioMed Central and take full advantage of:}

- Convenient online submission

- Thorough peer review

- No space constraints or color figure charges

- Immediate publication on acceptance

- Inclusion in PubMed, CAS, Scopus and Google Scholar

- Research which is freely available for redistribution 